\title{
Déterminants de la consommation de lait frais pasteurisé local à Ouagadougou au Burkina Faso
}

\author{
S. Ouedraogo ${ }^{1 *}$ H. Doanio ${ }^{2}$
}

Mots-clés

Lait - Consommation des ménages Modèle économétrique - Revenu Burkina Faso.

\begin{abstract}
Résumé
L'objectif de cette étude a été d'établir les déterminants de la consommation de lait frais pasteurisé dans la ville d'Ouagadougou au Burkina Faso. Une enquête rétrospective en un seul passage a été faite auprès de 120 ménages selon un échantillonnage semi-raisonné. Pour analyser les données, deux modèles économétriques ont été utilisés : le modèle " probit » pour estimer la probabilité de consommation de lait frais pasteurisé et le modèle linéaire simple d'Heckman pour estimer le niveau des dépenses consacrées à la consommation de ce produit. Les résultats ont montré que, parmi les variables étudiées, les principaux facteurs qui ont influencé la décision de consommer du lait frais pasteurisé et le niveau de consommation ont été le goût, la disponibilité, le revenu et la taille du ménage. Le revenu a été la principale contrainte à la consommation de lait frais pasteurisé. Par ailleurs, l'étude a souligné que le lait frais pasteurisé et le lait UHT importé étaient des produits "de luxe » consommés surtout par les ménages à revenu élevé. En revanche, le lait concentré et le lait caillé étaient consommés en majorité par les ménages à revenu faible. La consommation de yaourt et de lait en poudre a semblé moins dépendante du niveau de revenu. Pour améliorer la présence du lait frais local sur le marché d'Ouagadougou, les transformateurs doivent veiller à fournir un lait de bonne qualité (dont le goût est réputé supérieur) et à le rendre le plus accessible possible, en particulier dans les quartiers à revenus élevés.
\end{abstract}

\section{INTRODUCTION}

Le Burkina Faso figure parmi les plus grands importateurs de lait et produits laitiers en Afrique subsaharienne (10). Malgré l'importance du rôle joué par l'élevage dans l'économie et dans la réduction de la pauvreté, ce secteur ne couvre pas les besoins du pays en lait et produits laitiers. En effet, la production nationale a été estimée en 2000 à 171000 t d'équivalent lait (12) contre une demande d'environ $200000 \mathrm{t}$.

Ce déficit est compensé par des importations de produits laitiers essentiellement constituées de lait en poudre et de lait concentré.

1. Cnrst/Inera, BP 476, Ouagadougou, Burkina Faso.

Tél. : +22650319238; +22670278473

Fax : +22650340271; e-mail : ouedsouley@hotmail.com

2. Cifoeb, Ouagadougou, Burkina Faso.

* Auteur pour la correspondance
La FAO (4) estime à 85 p. 100 la part des importations dans la consommation des produits laitiers à Ouagadougou. Ces produits laitiers importés sont constitués à plus de 50 p. 100 de lait concentré et, dans une moindre mesure, de lait en poudre (4). Les importations laitières au Burkina Faso se sont accrues, surtout pendant les cinq dernières années, passant de 5,6 millions de Fcfa en 2002 à plus de 7 millions de Fcfa en 2004, et 6,4 millions de Fcfa en 2005 (3).

De nos jours, les produits laitiers occupent une place de plus en plus importante dans la consommation alimentaire, surtout en milieu urbain et particulièrement dans la ville d'Ouagadougou.

Le problème qui se pose aux décideurs est de pouvoir réduire les importations tout en assurant la satisfaction des besoins en lait et en produits laitiers des populations. Pour résoudre ce problème, des actions ont été entreprises à partir des années 1990, comme la création d'une concertation filière lait en vue de promouvoir la production laitière locale, la création du Programme national pilote 
de développement laitier en 1993, la création du ministère des Ressources animales en juin 1997, et la tenue de l'atelier national sur la politique laitière en juin 1998.

A cela s'est ajoutée la mise en place de plusieurs projets et microprojets de développement laitier pour permettre l'émergence du secteur laitier local. Des acteurs privés disposant de moyens substantiels se sont également impliqués dans la production de produits laitiers (14). Ces actions ont eu un impact sur la structuration des filières domestiques. Le secteur laitier local a connu un certain dynamisme avec l'apparition de plusieurs minilaiteries après plus d'une décennie d'actions gouvernementales.

Cependant, les unités locales de production et de transformation laitières se trouvent aujourd'hui en difficulté face à la concurrence des produits laitiers en provenance des pays industrialisés, essentiellement de l'Union européenne. Les contraintes de la filière locale sont liées en partie à un manque de connaissances du marché du lait et des produits laitiers qui pourraient permettre aux décideurs d'engager des actions pour que les produits laitiers locaux puissent reconquérir les marchés urbains.

Très peu d'études ont été faites en la matière au Burkina Faso, et celles qui existent sont souvent contradictoires. Pour certains auteurs, la production laitière locale est insuffisante et la majorité de la demande laitière locale ne peut être couverte qu'à partir d'importations (10). Pour d'autres, les importations ont un impact négatif sur la production locale de lait, parce que les produits laitiers importés à bas prix concurrencent de manière « déloyale » les produits laitiers locaux $(1,2,13)$.

Pour promouvoir le lait local et le rendre plus compétitif, il est primordial de connaître les déterminants de la consommation des produits laitiers à Ouagadougou. Il convient en effet de savoir quelles sont les relations de concurrence ou de complémentarité entre produits locaux et produits importés, d'évaluer les quantités consommées et de connaître les attentes des consommateurs pour les produits laitiers. En particulier, il est nécessaire de mieux comprendre les déterminants de la consommation de lait frais pasteurisé local qui représente une des formes essentielles de valorisation commerciale du lait local.

L'objectif de cette recherche a été d'analyser les déterminants de la consommation de lait frais pasteurisé à Ouagadougou. La capitale constitue le plus grand pôle de consommation du Burkina Faso, offrant un débouché majeur pour les produits laitiers locaux et importés. Le lait frais pasteurisé local est un lait chauffé, puis rapidement refroidi, selon des procédés mis en place localement dans les unités de transformation artisanales et semi-industrielles. Il est produit exclusivement à partir de lait de vache collecté au Burkina Faso. Le lait frais pasteurisé est essentiellement consommé sous forme de boisson rafraîchissante à différents moments de la journée.

\section{METHODOLOGIE}

Pour atteindre l'objectif de recherche, les auteurs ont utilisé deux modèles économétriques. Le modèle « probit » pour analyser la décision de consommation de lait frais pasteurisé et le modèle linéaire à deux étapes d'Heckman pour analyser le niveau des dépenses de consommation.

\section{Choix de deux modèles économétriques}

Par modèle économétrique, on entend une représentation simplifiée des lois gouvernant une ou plusieurs variables par un système cohérent de relations mathématiques. L'utilisation d'un modèle suppose au préalable une identification des variables et une spécification du modèle.

L'analyse économétrique permet d'affiner des relations entre une ou plusieurs variables dépendantes et d'autres variables à travers des fonctions. Pour l'analyse de la consommation de lait frais pasteurisé, les modèles linéaires simples Probit, Logit, ou Tobit peuvent être utilisés. Les modèles linéaires simples analysent l'influence des facteurs déterminant le niveau de la consommation de lait frais pasteurisé. Les modèles Probit et Logit se limitent à l'analyse de la décision de consommation, c'est-à-dire à l'analyse de l'influence des facteurs déterminant la probabilité de consommer, sans aborder l'intensité de la consommation de lait frais pasteurisé. Le modèle Tobit, quant à lui, permet d'analyser les facteurs influençant le niveau de consommation.

Il a été utilisé pour la présente étude, car l'objectif poursuivi était non seulement d'identifier les déterminants de la consommation de lait frais pasteurisé, mais aussi d'expliquer le niveau de consommation de celui-ci. L'estimation du modèle Tobit peut se faire selon deux méthodes : la méthode du maximum de vraisemblance et la méthode à deux étapes.

La méthode du maximum de vraisemblance suppose une simultanéité entre la décision de consommer et la détermination du niveau de consommation. Cela implique que les variables jugées significatives le sont en même temps pour la décision de consommation et pour le niveau de consommation. L'application de cette méthode sur le modèle Tobit donne des estimateurs sans biais et convergents.

La méthode à deux étapes permet de séparer de façon séquentielle la décision de consommation et le niveau de consommation. La probabilité de consommation est estimée, dans un premier temps, en utilisant un modèle Probit, puis le niveau de consommation est estimé, dans un second temps, en appliquant la méthode des moindres carrés sur un modèle linéaire. L'estimation de ces modèles linéaires par la méthode des moindres carrés ordinaires fournit aussi des estimateurs non biaisés et convergents. Cependant, l'estimation du modèle Tobit Heckman par la méthode à deux étapes a fait l'objet de critique dans la mesure où il y a souvent simultanéité entre la décision de consommation de lait frais local et la détermination du niveau de consommation de celui-ci.

Les variables déterminant la décision de consommation de lait frais local pasteurisé ont été différentes des variables déterminant le niveau de consommation de lait frais local pasteurisé. La méthode de vraisemblance n'était donc pas la mieux indiquée pour l'analyse de la consommation de lait frais local pasteurisé. Les auteurs ont ainsi utilisé la méthode Heckman à deux étapes pour l'estimation du modèle Tobit, ce qui a permis d'éviter les risques de biais de sélection. Il s'agissait d'établir les modèles économétriques expliquant, d'une part, le choix pour un individu de consommer ou non le lait frais pasteurisé et, d'autre part, le niveau individuel de consommation de lait frais pasteurisé.

Les auteurs ont fait l'hypothèse que la décision de consommer était uniquement liée à trois paramètres appréciés par le consommateur : le goût, la qualité et l'accessibilité du produit. En d'autres termes, la décision de consommation n'était ni liée à des paramètres internes aux ménages (nombre de personnes, niveau de revenu par exemple), ni au prix du produit. Ces paramètres ont en revanche été pris en compte dans l'explication des quantités consommées.

Pour la spécification des fonctions de consommation de lait frais pasteurisé, une analyse économétrique a été menée, permettant de cerner l'impact des variables suivantes : revenu, prix des produits laitiers importés (lait en poudre et lait concentré), prix du lait frais local pasteurisé et nombre de personnes vivant dans le ménage, sur 
le niveau de la consommation de lait pasteurisé quand un individu décidait de consommer.

\section{Choix de l'échantillonnage et collecte des données}

Les données primaires ont été collectées auprès de 120 ménages à Ouagadougou. L'échantillonnage a été fait selon un sondage aléatoire, en tenant compte de la démographie des quartiers, pour déterminer la répartition spatiale des habitants au regard des données de l'Institut national de la statistique et de la démographie (Insd) sur la population d'Ouagadougou. L'échantillon enquêté comprenait 60 ménages vivant dans les quartiers populaires (Zogona), 40 ménages vivant dans les quartiers résidentiels à moyen standing (1 200 logements) et 20 ménages vivant dans les quartiers résidentiels à haut standing. L'unité d'observation a été le ménage.

Les questionnaires d'enquête administrés aux différents ménages pendant une période de six mois ont servi de base à la collecte d'informations quantitatives et qualitatives relatives aux caractéristiques des ménages en matière de consommation des produits laitiers. Les questions ont porté à la fois sur les quantités consommées à l'échelle du ménage et sur les déterminants du choix du ménage. L'administration du questionnaire a été faite selon quatre axes principaux :

- les renseignements généraux sur le ménage (quartier, nombre de personnes, âge, ethnie, revenu, profession) ;

- la consommation de lait et produits laitiers du mois (type, fréquence, quantités achetées selon la saison, prix, lieu d'achat) ;

- les critères de consommation et de non-consommation ;

- les modes de consommation.

Les données secondaires ont été obtenues grâce à divers documents portant sur les études économiques de la filière lait, provenant principalement de l'Organisation des Nations unies pour l'alimentation et l'agriculture (FAO), du Centre international de recherche-développement sur l'élevage en zones subhumides (Cirdes), de l'Institut national de l'environnement et des recherches agricoles (Inera), et du ministère des Ressources animales. Les données de l'enquête ont été saisies à l'aide du logiciel Excel et les estimations économétriques ont été réalisées avec le logiciel Stata $(7,9)$.

\section{Spécification des modèles}

Deux modèles économétriques ont été spécifiés : le modèle probit pour estimer la probabilité de consommation de lait frais pasteurisé, et le modèle linéaire simple d'Heckman pour estimer le niveau des dépenses consacrées à la consommation de lait frais pasteurisé. L'estimation séquentielle de ces deux modèles a donné le modèle Tobit Heckman à deux étapes $(5,6)$.

\section{Description du modèle Probit}

La structure générale de ces modèles a modélisé la probabilité de choix et non le phénomène lui-même (la consommation de lait frais pasteurisé), permettant d'analyser la probabilité pour que le phénomène se réalise. Soit le choix consistant à consommer du lait frais pasteurisé ou non :

$\mathrm{Y}_{i}=\frac{1}{0}$ si l'individu consomme le lait frais

Sinon $\mathrm{Y}_{i}$ est la décision de consommer du lait frais pasteurisé.

On a :

$\mathrm{P}_{i}=\mathrm{P}\left(\mathrm{Y}_{i}=1\right)$

Ce modèle admet pour variable expliquée la probabilité de consommation de lait frais, conditionnellement aux variables exogènes. Ainsi, on considère le modèle suivant : $\mathrm{p}_{\mathrm{i}}=\operatorname{prob}\left(\mathrm{y}_{\mathrm{i}}=1 / \mathrm{x}_{\mathrm{i}}\right)=\mathrm{F}\left(\mathrm{x}_{\mathrm{i}} \beta\right)$ pour tout $\mathrm{i}=1 \ldots \mathrm{N}$

où la fonction $\mathrm{F}(\mathrm{.}$ ) désigne une fonction de répartition.

Dans le cas présent, cette fonction est représentée par la fonction de répartition de la loi normale centrée réduite pour tout $\omega \in \mathrm{R}$ :

$\mathrm{F}(\omega)=\int_{-\infty}^{\omega} \frac{1}{\sqrt{ } 2 \Pi} \mathrm{e}^{-\frac{z^{2}}{2}} \mathrm{dz}=\Phi(\omega)$

$\mathrm{P}_{i}$ est expliquée par un ensemble de caractéristiques du lait frais pasteurisé.

Soit la matrice $\mathrm{x}\left(\mathrm{x}_{1} ; \mathrm{x}_{2} ; \ldots \mathrm{x}_{\mathrm{k}}\right)$ l'ensemble de ces caractéristiques ; alors $\mathrm{P}_{i}=\mathrm{F}\left(\beta^{\prime} \mathrm{x}_{\mathrm{i}}\right)$ avec $\mathrm{F}$ une fonction à déterminer en définissant $\mathrm{F}\left(\beta^{\prime} x_{i}\right)$ comme la valeur de la fonction de répartition de la loi normale centrée réduite $\mathrm{N}(0,1)$ considérée au point $\mathrm{x}_{\mathrm{i}} \beta$. Cela implique que :

$\mathrm{P}_{\mathrm{i}}=\Phi\left(\mathrm{x}_{\mathrm{i}} \beta\right)=\int_{-\infty}^{x i \beta} \frac{1}{\sqrt{ } 2 \Pi} \mathrm{e}^{-\frac{\mathrm{z}^{2}}{2}} \mathrm{dz} \quad \forall \mathrm{i}=1 \ldots \mathrm{N}$

Ceci est donc le modèle Probit appliqué à l'étude pour déterminer les critères de choix et de non-choix des consommateurs de lait frais pasteurisé.

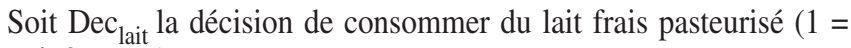
oui, $0=$ non)

$\mathrm{P}_{\text {lait }}$ représente la probabilité que le ménage se décide à consommer du lait frais

avec $P_{\text {lait }}=f$ (goût, qual, accès)

où goût représente l'appréciation du goût du lait par le ménage (1 = très mauvais, $2=$ mauvais, $3=$ bien, $4=$ très bien, $5=$ excellent) ; qual l'appréciation de la qualité du lait frais par le ménage $(1=$ très mauvais, ... 5 = excellent) ; accès l'appréciation de l'accessibilité du lait frais par le ménage ( $1=$ très mauvais, $\ldots 5=$ excellent $)$.

\section{Description du modèle linéaire simple}

Soit $\left(\mathrm{C}_{\text {lait }}\right)$ le niveau de consommation individuelle de lait frais pasteurisé

avec $\mathrm{C}_{\text {lait }}=\mathrm{f}\left(\mathrm{rev}, \mathrm{P}_{1}, \mathrm{P}_{\mathrm{lc}}, \mathrm{P}_{\mathrm{lp}}\right.$, nombre $)$

où $C_{\text {lait }}$ représente le niveau de consommation mensuel du lait frais local pasteurisé du ménage (en francs CFA) ; rev le revenu mensuel du ménage (Fcfa) ; $P_{l}$ l'appréciation du prix du lait frais pasteurisé par les différents consommateurs $(1=$ peu cher, 2 = acceptable, $3=$ trop cher); $P_{l c}$ l'appréciation du prix du lait concentré par les différents consommateurs $(1=$ peu cher, $\ldots 3=$ trop cher $) ; P_{l p}$ l'appréciation du prix du lait en poudre par les différents consommateurs ( 1 = peu cher, ... $3=$ trop cher) ; nombre le nombre de personnes dans le ménage.

La variable dépendante est en valeur. Les variables explicatives, revenu et nombre, sont quantitatives et le reste des variables multinomiales.

Pour la spécification du modèle concernant le niveau de la consommation de lait frais pasteurisé, la fonction linéaire a été retenue. Cette forme fonctionnelle est conforme à la théorie keynésienne de la consommation. Les données de l'étude étant en coupes instantanées, la constance de l'élasticité de la demande par rapport à toutes les variables explicatives montre que cette forme ne prend pas en compte l'adaptation du comportement des consommateurs. Mais cela ne peut être considéré comme un inconvénient, au vu de la nature des données.

On a :

$\mathrm{C}_{\text {lait }}=\alpha+\beta_{1}$ rev $+\beta_{2} \mathrm{P}_{1}+\beta_{3} \mathrm{P}_{\mathrm{lc}}+\beta_{4} \mathrm{P}_{1 \mathrm{p}}+\beta_{5}$ nombre $+\varepsilon_{\mathrm{i}}$ 
Ce modèle économétrique linéaire simple ainsi défini s'insère dans le modèle linéaire général basé sur six hypothèses : $\mathrm{H} 1$, le modèle est correctement spécifié ; H2, la moyenne des erreurs est nulle ; H3, la variance des erreurs est constante ; H4, il y a absence d'autocorrélation des erreurs, c'est-à-dire $\varepsilon_{\mathrm{i}}$ et $\varepsilon_{\mathrm{j}}$ sont indépendants pour i différent de j ; H5, il n'y a pas de relation linéaire exacte entre les variables explicatives ; H6, il y a indépendance entre les erreurs $\varepsilon_{\mathrm{i}}$ et les variables explicatives $\mathrm{x}_{\mathrm{ij}}$.

Avec $\varepsilon$ le terme d'erreur. L'estimation de la fonction se fera par la méthode des moindres carrés ordinaires avec le logiciel Stata. Le tableau I présente les signes théoriques attendus des variables explicatives.

\section{RESULTATS ET DISCUSSION}

\section{Consommation des produits laitiers dans les ménages}

La consommation moyenne par individu a été de $124 \mathrm{~kg} / \mathrm{an}$ et de $371 \mathrm{~kg} /$ ménage (tableau II), équivalente à une dépense moyenne annuelle de $65500 \mathrm{Fcfa} / \mathrm{individu}$ et de $195850 \mathrm{Fcfa} / \mathrm{ménage}$. Ces moyennes ne mettent pas en avant de très fortes variations entre ménages à faible revenu et ménages aisés. Elles étaient, par ailleurs, nettement supérieures aux moyennes nationales. La FAO (4) estime à $30 \mathrm{~kg} / \mathrm{an} /$ personne la consommation moyenne de lait et produits laitiers au Burkina Faso.

Si l'on considère les quantités (en kilogrammes équivalents lait), le lait en poudre et le lait frais pasteurisé ont été de loin les produits les plus consommés. Ces produits ont représenté chacun

\section{Tableau I}

Signes théoriques des différentes variables explicatives

\begin{tabular}{lcc} 
Variable explicative & $\begin{array}{c}\text { Coefficient } \\
\text { des variables }\end{array}$ & $\begin{array}{c}\text { Signe théorique } \\
\text { attendu }\end{array}$ \\
\hline Revenu (rev) & $\beta 1$ & + \\
$\begin{array}{l}\text { Prix du lait frais pasteurisé (Pl) } \\
\text { Prix du lait concentré (Plc) }\end{array}$ & $\beta 2$ & - \\
$\begin{array}{l}\text { Prix du lait concentré } \\
\text { en poudre (Plp ) }\end{array}$ & $\beta 3$ & + \\
Nombre & $\beta 4$ & + \\
& $\beta 5$ & -
\end{tabular}

27 p. 100 de la consommation totale des ménages, suivis du beurre (17 p. 100), du lait UHT (12 p. 100) et des yaourts (10 p. 100). En revanche, en valeur, le lait frais pasteurisé a représenté 29 p. 100 des dépenses de consommation, les yaourts 25 p. 100 et le lait en poudre 18 p. 100. Le lait frais pasteurisé a donc été le premier produit laitier de consommation d'Ouagadougou.

L'analyse de la structure de consommation par type de produit a fait ressortir des variations sensibles en fonction du niveau de revenu. La figure 1 montre que le lait frais pasteurisé et le lait UHT étaient consommés par les ménages aisés, tandis que le lait concentré et le lait caillé étaient consommés par les ménages les plus pauvres. La consommation de yaourt et de lait en poudre n'a pas semblé être fortement influencée par le niveau de revenu.

Dans l'ensemble, la consommation de produits laitiers a été très fortement dépendante du niveau de revenu des ménages. La même constatation a été faite par le ministère délégué chargé des Ressources animales (Mdcra) (11) qui rapporte que seulement 20 p. 100 des enfants des familles dont le revenu mensuel était inférieur à 50000 Fcfa consommaient des produits laitiers, contre plus de 80 p. 100 dans les familles dont le revenu mensuel était supérieur à $150000 \mathrm{Fcfa}$. Par ailleurs, le lait frais pasteurisé et le lait UHT étaient considérés comme des produits de luxe par les habitants d'Ouagadougou.

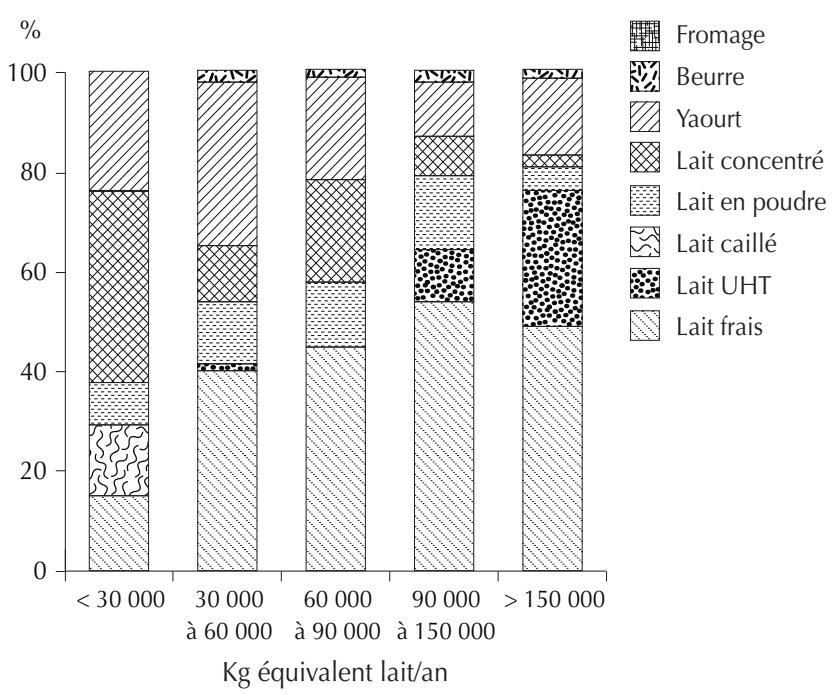

Figure 1 : consommation des différents produits laitiers dans les ménages par classe de revenu.

Tableau II

Consommation totale de produits laitiers dans les ménages et par individu selon la classe de revenus des ménages à Ouagadougou

\begin{tabular}{|c|c|c|c|c|c|c|c|}
\hline Revenu estimé & Nb. ménages & $\begin{array}{l}\text { Taille moyenne } \\
\text { des ménages }\end{array}$ & ET & $\begin{array}{c}\text { Conso/ménage } \\
\text { (kg/an) }\end{array}$ & ET & $\begin{array}{c}\text { Conso/individu } \\
\text { (kg/an) }\end{array}$ & ET \\
\hline$<30000 \mathrm{Fcfa} / \mathrm{mois}$ & 33 & 7 & 3 & 21,5 & 36 & 7,15 & 12 \\
\hline 30000 à $60000 \mathrm{Fcfa} / \mathrm{mois}$ & 40 & 4 & 3 & 62 & 111 & 20,69 & 37,24 \\
\hline 60000 à 90000 Fcfa/mois & 15 & 5 & 2 & 100 & 168,8 & 33,24 & 56,27 \\
\hline 90000 à $150000 \mathrm{Fcfa} /$ mois & 18 & 6 & 3 & 215 & 175 & 71,68 & 58,47 \\
\hline$>150000 \mathrm{Fcfa} / \mathrm{mois}$ & 14 & 5 & 2 & 951 & 775 & 317 & 258,52 \\
\hline Total & 120 & 7 & 4 & 371 & 1978 & 124 & 659,55 \\
\hline
\end{tabular}

Source : données d'enquête ; ET : écart-type ; Conso : consommation 


\section{Analyse des déterminants de la décision de consommation de lait frais pasteurisé (modèle probit)}

\section{Validité économétrique du modèle Probit}

Les résultats de l'estimation du modèle Probit sont présentés dans le tableau III. Pour tester la signification d'ensemble, les auteurs ont utilisé la probabilité de signification du test de Hosmer-Lemeshow et le pourcentage de prédictions fausses. Le test de HosmerLemeshow permet de tester l'hypothèse nulle que l'ajustement est bon contre l'hypothèse alternative que l'ajustement est mauvais. Le pourcentage de prédictions fausses montre l'erreur de prédiction du modèle.

La probabilité de signification du test de Hosmer-Lemeshow a été de 0,0563 , soit 5,63 p. 100. Supérieur à 5 p. 100, l'ajustement au seuil de 5 p. 100 était donc bon. Cependant il faut émettre des réserves car la valeur du test de Hosmer-Lemeshow était assez élevée $(15,14)$, impliquant qu'il y avait un écart non négligeable entre les valeurs observées et les valeurs prédites.

Le pourcentage de prédictions fausses du modèle a été de 32,23 p. 100, soit, si l'on considère la consommation de lait frais pasteurisé par cent personnes, elle a été mal prédite par le modèle pour 32 d'entre elles. Ce pourcentage peut cependant être jugé acceptable.

\section{Signification statistique des coefficients individuels et implications économiques}

La probabilité de signification de chaque paramètre (goût, qualité et accès) a permis de tester l'hypothèse nulle que le paramètre estimé était nul pour chaque coefficient, contre l'hypothèse alternative qu'il était différent de zéro.

Le goût et l'accessibilité (tels qu'ils étaient perçus par les consommateurs) ont eu un effet positif sur la probabilité de consommation de lait frais (tableau IV). En d'autres termes, la probabilité de consommer du lait frais pasteurisé a augmenté quand le goût était plus apprécié par les consommateurs et/ou quand l'accessibilité était jugée meilleure. Ces deux variables ont eu des coefficients statistiquement significatifs d'après les résultats de l'estimation.

Le goût du lait frais pasteurisé est naturel mais pas très sucré. Or, la plupart des ménages ont préféré les produits laitiers sucrés. Le goût a été ainsi un élément déterminant dans la décision de consommation de lait frais pasteurisé. Pour en augmenter la consommation, les transformateurs doivent veiller à fournir un lait de bonne qualité (dont le goût est réputé supérieur) et le plus accessible possible, en particulier dans les quartiers à revenus élevés.

\section{Tableau III}

Résultats du modèle probit

\begin{tabular}{lcccc} 
Variable & Coefficient & ES & t-statistic & Probabilité \\
Goût & 0,2709364 & 0,1336653 & 2,41 & 0,016 \\
Qualité & $-0,1017249$ & 0,1382996 & $-0,83$ & 0,409 \\
Accès & 0,3337339 & 0,1550918 & 2,37 & 0,018 \\
\hline H-L statistic : 15,14 & \multicolumn{3}{c}{ Prob[Chi-Sq (8 df)] : 0,0563 } \\
Andrews statistic : 14,48 & \multicolumn{2}{c}{ Prob[Chi-Sq (10 df)] : 0,1520 }
\end{tabular}

Source : données d'enquête ; ES : erreur standard
L'accessibilité traduit les coûts supplémentaires qu'un ménage doit supporter pour pouvoir trouver le produit sur le marché. A Ouagadougou les points de vente de lait frais pasteurisé étaient surtout concentrés dans le centre ville, plutôt que dans les quartiers périphériques. Par ailleurs, la saisonnalité de la production de lait a constitué la principale contrainte dans la mesure où elle n'a pas permis de stabiliser l'offre et de fidéliser les consommateurs. Lorsque la distance à parcourir était moins grande pour trouver du lait frais pasteurisé, les coûts de transaction diminuaient, avec pour effet d'inciter les ménages à en consommer. La proximité a augmenté la probabilité de consommation de lait frais pasteurisé de 0,1260 .

La qualité, telle qu'elle était appréciée par les consommateurs, a eu un impact négatif sur la probabilité de consommation de lait frais pasteurisé. La qualité du lait frais pasteurisé a semblé être appréciée positivement par l'ensemble des ménages consommateurs et non-consommateurs, expliquant pourquoi cette variable n'était pas statistiquement significative dans la probabilité de consommation de lait frais pasteurisé.

\section{Analyse du niveau de dépenses allouées à la consommation de lait frais (modèle linéaire par la méthode d'Heckman)}

\section{Validité économétrique du modèle linéaire}

Les résultats de l'estimation du modèle linéaire par la méthode d'Heckman sont consignés dans le tableau V. La validité d'ensemble du modèle estimé a été analysée à l'aide de la valeur du coefficient de détermination $\mathrm{R}^{2}$ ajusté et de la statistique chi $^{2}$ de Wald. La méthode d'estimation a été celle de Heckman à deux étapes pour corriger les risques de biais de sélection. La statistique $\mathrm{R}^{2}$ ajustée qui mesure l'adéquation du modèle a été acceptable, surtout du point de vue de la forme fonctionnelle. Dans le cas de l'étude, le coefficient $\mathrm{R}^{2}$ ajusté a été de 56,67 p. 100, indiquant que le niveau des dépenses consacrées à la consommation de lait frais a été expliqué à 56,67 p. 100 par les variables indépendantes incluses dans le modèle.

Un autre indicateur de l'adéquation d'ensemble du modèle a été la statistique chi $^{2}$ de Wald. Cette statistique teste l'hypothèse nulle que tous les paramètres estimés sont simultanément nuls contre l'hypothèse alternative qu'au moins un de ces paramètres n'est pas nul. Dans le cas de l'étude, la probabilité $\left(\mathrm{chi}^{2}\right)$ a été de 0,000 , donc inférieure au seuil de signification de 1 p. 100. Le seuil de signification indique la probabilité d'erreur commise en affirmant que tous les paramètres sont différents de zéro alors qu'ils ne le sont pas. Ici on rejette l'hypothèse que les paramètres sont conjointement nuls. Le coefficient du ratio de Mills n'a pas été significatif (prob $=0,65)$. Ce qui implique que la méthode des moindres carrés ordinaires pouvait être utilisée pour l'estimation du modèle.

\section{Tableau IV}

Effets marginaux

\begin{tabular}{lcrrr} 
Variable & $\begin{array}{c}\text { Effet marginal } \\
(\mathbf{d y} / \mathbf{d x})\end{array}$ & ES & t-statistic & Probabilité \\
\hline Goût & 0,1023 & 0,0422 & 2,43 & 0,015 \\
Qualité & $-0,0384$ & 0,0464 & $-0,83$ & 0,408 \\
Accès & 0,1261 & 0,0532 & 2,37 & 0,018
\end{tabular}

Source : données d'enquête ; ES : erreur standard 
Tableau V

Résultat du modèle linéaire d'Heckman

\begin{tabular}{|c|c|c|c|c|}
\hline Variable & Coefficient & ES & t-statistic & Probabilité \\
\hline Revenu & 0,1847 & 0,3254 & 5,68 & 0,000 \\
\hline Prix lait frais pasteurisé & 457,5632 & 3203,423 & 0,14 & 0,886 \\
\hline Prix lait concentré & $-1036,294$ & 3536,054 & $-0,29$ & 0,769 \\
\hline Prix lait en poudre & $-4066,407$ & 3349,799 & $-1,21$ & 0,225 \\
\hline Nb. de personnes (taille du ménage) & $-2225,439$ & 1073,057 & $-2,07$ & 0,038 \\
\hline$R^{2}=61,47 \%$ & \multirow{2}{*}{$\begin{array}{l}\text { ratio de Mills }=3587,9216 \\
\text { prob(Mills) }=0,65\end{array}$} & & & \\
\hline$R^{2}=56,67 \%$ & & & & \\
\hline \multirow{2}{*}{\multicolumn{2}{|c|}{$\begin{array}{l}\text { Wald } \operatorname{chi}^{2}(5)=73,36 \\
\text { Prob chi }{ }^{2}=0,0000\end{array}$}} & & & \\
\hline & & & & \\
\hline
\end{tabular}

Source : données d'enquête ; ES : erreur standard

\section{Signification statistique des coefficients individuels et implications économiques}

Le revenu a exercé un effet positif sur le niveau de dépense en lait frais pasteurisé des ménages. Les tests ont montré que le coefficient de cette variable était statistiquement significatif, confirmant la théorie économique selon laquelle la consommation d'un produit est essentiellement dépendante du revenu. Une augmentation du revenu d'un franc CFA a entraîné une augmentation des dépenses de consommation en lait frais de 0,1846 Fcfa (tableau VI). L'élasticité du revenu a été de 4,63. Une augmentation du revenu a entraîné une augmentation plus que proportionnelle des dépenses de consommation de lait frais pasteurisé. Le lait frais pasteurisé était donc un produit réservé aux ménages à revenu élevé.

Le nombre de personnes dans le ménage (taille du ménage) a influencé négativement le niveau des dépenses de consommation du ménage en lait frais. Une augmentation d'une personne dans un ménage a entraîné une baisse des dépenses de consommation de lait frais de $2225 \mathrm{Fcfa} /$ mois. L'élasticité de la consommation par rapport au nombre de personnes dans le ménage a été de $-1,71$. Ainsi, plus le nombre de personnes dans un ménage augmentait et moins la disponibilité financière pour la consommation de lait frais pasteurisé était élevée. Ce produit était donc réservé aux ménages à nombre de personnes réduit.

Le prix du lait frais pasteurisé, le prix du lait concentré et le prix du lait en poudre ont eu des coefficients non significatifs. Le prix

\section{Tableau VI}

Elasticité des différentes variables explicatives

\begin{tabular}{|c|c|c|c|c|}
\hline Variable & $\begin{array}{c}\text { Elasticité } \\
(\text { ey/ex })\end{array}$ & ES & $\mathbf{z}$ & probabilité \\
\hline Revenu & 4,63 & 12,43 & 0,37 & 0,70 \\
\hline $\begin{array}{l}\text { Prix lait frais } \\
\text { pasteurisé }\end{array}$ & 0,39 & 2,71 & 0,15 & 0,88 \\
\hline Prix lait concentré & $-0,87$ & 3,83 & $-0,23$ & 0,82 \\
\hline Prix lait en poudre & $-3,48$ & 10,01 & $-0,35$ & 0,72 \\
\hline Nb. de personnes & $-1,71$ & 4,66 & $-0,37$ & 0,71 \\
\hline
\end{tabular}

Source : données d'enquête : ES : erreur standard du lait frais pasteurisé a influencé positivement le niveau des dépenses en lait frais pasteurisé dans les ménages. Autrement dit, quand le prix du lait frais pasteurisé augmentait, la demande en lait frais baissait, ou quand les consommateurs trouvaient que le prix d'un produit était trop cher, ils baissaient leur consommation de ce produit. Cette variable n'a pas été significative car le niveau de consommation de lait frais de certains ménages n'était pas dépendant du prix. Le lait frais était d'abord consommé pour ses vertus nutritives et son goût. Lambey a montré que le prix était non seulement un indicateur du coût mais aussi une information utilisée par les individus pour évaluer la qualité d'un produit (8).

Tous les ménages qui consommaient beaucoup de lait frais pasteurisé consommaient aussi et en grande quantité du lait concentré et/ou en poudre. La réciproque était moins évidente : les coefficients d'impact des prix du lait concentré et du lait en poudre sur le niveau de dépense en consommation de lait frais n'ont pas été significatifs.

\section{CONCLUSION}

Les résultats obtenus avec le modèle probit et le modèle linéaire simple d'Heckman ont indiqué que le niveau de revenu, la taille du ménage, le goût et l'accessibilité étaient des variables déterminantes de la consommation de lait frais pasteurisé. Le niveau de revenu et la taille du ménage ont révélé une segmentation du marché du lait et des produits laitiers. D'une part, le lait frais pasteurisé et le lait UHT étaient des produits onéreux, consommés par les ménages aisés de taille réduite. D'autre part, le lait concentré et le lait caillé étaient des produits de grande consommation, consommés par l'ensemble des ménages, et en particulier par les ménages pauvres de grande taille. Le lait frais pasteurisé a donc semblé être un produit de luxe pour les habitants d'Ouagadougou.

\section{BIBLIOGRAPHIE}

1. AGECO, 2006. Analyse de la problématique de l'importation de la poudre de lait au Burkina Faso et de son effet sur le développement de la filière lait. Rapport de mission de D.M. Gouin et Y. Proulx. Quebec, Canada, Groupe Ageco, 69 p.

2. CORNIAUX C., NIAFO Y., POCCARD-CHAPUIS R., COULIBALY D., 2005. Consommation de lait et de produits laitiers dans les ménages de Ségou, Mali. Montpellier, France, Cirad, 21 p. 
3. DIRECTION DES DOUANES, 2006. Statistiques douanières 2006. Ouagadougou, Burkina Faso, ministère des Finances, 145 p.

4. FAO, 2003. Annuaire de la production et du commerce. www.fao.org (09.08.2005)

5. HECKMAN J., 1979. Sample selection bias as a specification error. Econometrica, 47: 153-161.

6. HURLIN C., 2003. Econométrie des variables qualitatives. Master économétrie et statistique appliquée, université d'Orléans, France, 59 p.

7. KPODAK K., 2005. Manuel d'initiation à Stata (vers. 8). ClermontFerrand, France, Cerdi, $77 \mathrm{p}$.

8. LAMBEY C., 2000. Price as an indicator of sacrifice. Le prix dans son rôle négatif. In : Tendenze del marketing in Europa. Venezia, Italia, Universita Ca'Foscani, 28 p.

9. OUELLET S., LEBLOND S., BELLEY-FERRY I., 2005. Guide d'économétrie appliquée pour Stata. Montréal, Canada, université de Montréal, dépt ECN, 69 p.
10. METZER R., CENTRES J.M., THOMAS L., LAMBERT J.C., 1995. L'approvisionnement des villes africaines en lait et produits laitiers. Rome, Italie, FAO, 102 p. (Production et santé animale $n^{\circ} 124$ )

11. MDCRA, 1995. Etude des marchés du lait et des produits laitiers d'Ouagadougou et de Bobo Dioulasso. Rapport provisoire. Ouagadougou, Burkina Faso, Mdcra.

12. MRA, 2001. Plan d'actions et programme d'investissement du secteur de l'élevage au Burkina Faso. Ouagadougou, Burkina Faso, MRA.

13. OUDET M., 2005. La révolution blanche est-elle possible au Burkina Faso, et plus largement en Afrique de l'Ouest ? Koudougou, Burkina Faso, Misereor, $30 \mathrm{p}$

14. SANON Y., 2006. Analyse de la filière laitière locale burkinabé : les acteurs et leurs organisations dans la périphérie de Bobo-Dioulasso et d'Ouagadougou. In : atelier Vers de nouvelles politiques laitières, Bamako, Mali, 29 mai - 2 juin 2006.

Accepté le 06.02.2010

\section{Summary}

Ouedraogo S., Doanio H. Determinants of the Consumption of Local Fresh Pasteurized Milk in Ouagadougou

The objective of this paper was to establish the determinants of the consumption of fresh pasteurized milk in the city of Ouagadougou in Burkina Faso. A one-time retrospective survey was conducted in 120 households selected by stratified sampling. To analyze the results, two econometric models were used: the probit model to assess the probability of fresh pasteurized milk consumption, and Heckman's simple linear model to assess the level of expenses devoted to the consumption of this product. Results showed that, among the studied variables, the major factors that influenced the consumption of fresh pasteurized milk were taste, availability, income, and size of household. The income was the main constraint to the consumption of fresh pasteurized milk. The study also showed that fresh pasteurized milk and imported UHT milk were "luxury" products mainly consumed by high income households. On the other hand, condensed milk and local fermented milk were mostly consumed by low income households. The consumption of yoghurt and dry milk seemed to depend less on the income level. To increase the supply of fresh milk in Ouagadougou's markets, processors should propose a good quality product (i.e. with a recognized good taste), with high accessibility, in particular in high income districts.

Keywords: Milk - Household consumption - Econometric model - Income - Burkina Faso.

\section{Resumen}

Ouedraogo S., Doanio H. Determinantes del consumo de leche fresca pasteurizada localmente en la ciudad de Ouagadougou

El objetivo del presente artículo fue el de cernir las características del consumo de leche fresca pasteurizada en la ciudad de Ouagadougou, en Burkina Faso. En una sola visita, se realizó una encuesta retrospectiva de 120 hogares, según un muestreo semi estudiado. Para analizar los datos, se utilizaron dos modelos econométricos. El modelo "probit" para estimar la probabilidad de consumo de leche fresco pasteurizado y el modelo linear simple de Heckman para estimar el nivel de gastos dedicados al consumo de este producto. Los resultados mostraron que, entre las variables estudiadas, los principales factores que influenciaron la decisión de consumir leche fresca pasteurizada y el nivel de consumo fueron el gusto, la disponibilidad, el ingreso y el tamaño del hogar. El ingreso fue el principal obstáculo para el consumo de leche fresca pasteurizada. Por otro lado, el estudio subraya que la leche fresca pasteurizada y la leche UHT importada fueron los productos "de lujo" consumidos sobre todo por los hogares de ingreso elevado. La leche concentrada y la leche cortada, por el contrario, fueron consumidos por la mayoría de los hogares de ingreso bajo. El consumo de yogurt y de leche en polvo pareció menos dependiente del nivel de ingreso. Para mejorar la presencia de leche fresca local en el mercado de Ouagadougou, los transformadores deben proporcionar una leche de buena calidad (con gusto de reputación superior) y hacerlo lo más accesible posible, particularmente en los barrios de ingresos elevados.

Palabras clave: Leche - Consumo familiar - Modelo econométrico - Renta - Burkina Faso. 